\title{
Anti aquaporin 4 positive neuromyelitis optica spectrum disorder and primary Sjögren syndrome following successful therapy for chronic hepatitis C - report of a clinical case
}

\author{
Carmen Predoiu', Laura Dumitrescu', Adela Danau', Liviu Cozma', Irina Orban', \\ Antonia Lefter ${ }^{1}$, Adriana Nicolau ${ }^{2}$, Radu Tanasescu ${ }^{1,3}$ \\ ${ }^{1}$ Second Neurology Department, CDPC, Colentina Clinical Hospital, Bucharest, Romania \\ 2Internal Medicine Department, CDPC, Colentina Clinical Hospital, Bucharest, Romania \\ ${ }^{3}$ General Medical School, "Carol Davila" University of Medicine and Pharmacy, Bucharest, Romania
}

\begin{abstract}
Neuromyelitis optica spectrum disorders (NMOSD) are a heterogeneous group of potentially disabling central nervous system inflammatory disorders. Association with other organ-specific or multisystem autoimmune disorders is commonly encountered. We report the case of a Caucasian woman with AQP4-IgG positive NMOSD and primary Sjögren syndrome - previously reported as a poster at the 14th Congress of the Romanian Society of Neurology (abstract published in ROJN, Vol. XV, Suppl). The clinical onset followed the successful treatment of chronic hepatitis $\mathrm{C}$ with interferon alpha and ribavirin. To the best of our knowledge, similar clinical presentations have not been described. The etiopathogenic treatment included high dose methylprednisolone, immune suppression with cyclophosphamide, and periodic plasma exchanges. In spite of an active disease with very high baseline AQP4-IgG titres, during the four years of treatment the patient has had only one relapse and has no residual disability.
\end{abstract}

Keywords: neuromyelitis optica spectrum disorders, AQP4-IgG, Sjögren syndrome, chronic hepatitis $\mathrm{C}$, interferon alpha

\section{INTRODUCTION}

Neuromyelitis optica spectrum disorders (NMOSDs) are a heterogeneous group of potentially disabling central nervous system inflammatory diseases, classically defined by a commonly shared phenotype (i.e., neuromyelitis optica or Devic's syndrome) and recently reclassified according to their pathogenic mechanisms (1). Most NMOSDs are an autoimmune astrocytopathy mediated by pathogenic immunoglobulin $\mathrm{G}$ anti aquaporin 4 antibodies (AQP4-IgGs). AQP4-IgGs seronegative cases are typically associated with anti myelin oligodendrocyte glycoprotein antibodies (MOGIgGs); sometimes AQP1-IgGs or no disease-specific antibodies are found (2). The classical presentation is with relapsing, or rarely monophasic, optic nerve and spinal cord involvement. Since the discovery of AQP4-IgGs, several other highly characteristic clinical syndromes accompanied by specific magnetic resonance imaging (MRI) changes were described (i.e., area postrema syndrome, acute diencephalic syndromes, including narcolepsy and acute brainstem and cerebral syndromes). The selective involvement of these specific regions is closely related to the distribution / density of astrocytic AQP4 and/or to the topographical distribution of oligodendrocyte susceptibility to inflammation and local homeostatic changes. The latest diagnostic criteria (Wingerchuk, 2015) use specific combinations of clinical, serologic and central nervous system MRI findings for a positive diagnosis (1). 
The etiopathogenic treatment is mostly empirical, and includes high dose methylprednisolon and plasma exchanges or intravenous immunoglobulin for relapses, and chronic immunosuppression (e.g., azathyoprine and cyclophosphamide) for inducing remission and relapse prevention (3). Association with other organ-specific or multisystem autoimmune disorders, such as Sjögren syndrome, is relatively common, and co-occurrence of Sjögren syndrome has been reported in $15.7 \%$ of the cases, according to one review (4).

\section{CLINICAL CASE}

We report the case of a Caucasian woman with AQP4-IgG positive NMOSD and primary Sjögren syndrome. At age 53 she was diagnosed with chronic hepatitis $\mathrm{C}$ virus (HCV) infection. She received pegylated interferon alpha and ribavirin treatment for two years. The antiviral regimen resulted in sustained undetectable HCV viremia after the first 3 months. At age 55, several months after finishing the antiviral treatment, she experienced walking disturbances, which subsided spontaneously in approximately one week. A year later she had a similar self-limited episode. No medical assessment was performed. At age 57 she was evaluated in our Clinic for paroxysmal sensori-motor phenomena (paroxistic left lower limb paresthesia and right lower limb hypertonia triggered by right leg movement); at the time of the first examination the symptoms had been stable for several months and no central nervous system structural changes had been found at a recent 1.5 Tesla MRI, except for a few non-specific supratentorial white matter lesions. Shortly after the initial evaluation, the frequency of the paroxistic phenomena increased to hundreds per day; concomitantly the patient developed hypoesthesia below T5, and bilateral upper motor neuron syndrome in the lower limbs. The MRI showed signal changes compatible with longitudinally extensive T3-T5 transverse myelitis; a dural arteriovenous fistula was considered in the differential diagnosis, but the spinal angio-MRI found no suggestive sings. The neurological deficits reversed after 5 grams of methylprednisolone and the residual paroxysmal phenomena responded promptly to low doses of carbamazepine. The workup revealed high serum anti Ro antibodies (20 times higher than the upper reference limit), and minor salivary gland histopathological examination confirmed the diagnostic of Sjögren syndrome (in a paucisymptomatic patient, but with xerophtalmia confirmed several Schirmer tests). Serum AQP4-IgGs, blood - cerebrospinal fluid (CSF) immunoglobulin index, and CSF oligoclonal bands were considered for the differential diagnosis (relapsing space-limited neuromyelitis optica/NMOS versus relapsing-remitting multiple sclerosis), but these were postponed, considering the patient's option to monitor the clinical evolution prior to starting a chronic immunomodulatory/ immunosuppressive treatment. Crioglobulins and other relevant tests for chronic infections and autoimmune diseases were negative. After two years the patient developed another spinal cord relapse, promptly recovered after 5 grams methylprednisolone. Serum AQP4-IgGs came back in high positive titre and no intrathecal immunoglobulin synthesis was observed (128 times the upper reference range value). Two months latter another relapse occurred, resulting in paraparesis, urinary incontinence and lower limb paresthesia (Fig. 1). Plasma exchange and another 5 grams of methylprednisolon were administered. Immunosuppressive treatment with monthly cyclophosphamide and methylprednisolon pulses was started (700 mg/ $\mathrm{m}^{2} /$ month, and $1.5-3$ grams/month, respectively). The clinical picture slowly improved and AQP4IgGs titres declined (30 times the upper reference value). After five months the patient developed viral pneumonia complicated with bacterial lung infection and sepsis. While still in recovery, the patient developed an area postrema syndrome; AQP4-IgGs titre proved to be high once again (i.e., 100 times the upper reference range value), and the symptoms subsided after plasma exchange and medium dose corticosteroids. Considering that the patient continued to have frequent mild to moderate bacterial and viral infections, cyclophosphamide was replaced with monthly plasma exchanges (one and a half volumes per month) followed by methylprednisolone pulses (1.5 grams per month). The administration of hydroxicloroquine, an immunomodulatory drug initiated after the diagnosis of Sjögren syndrome, was continued. No exacerbations occurred and the AQP4-IgGs titre decreased once again. After 8 months the patient was switched on azathyoprine. Over the following year, no other 


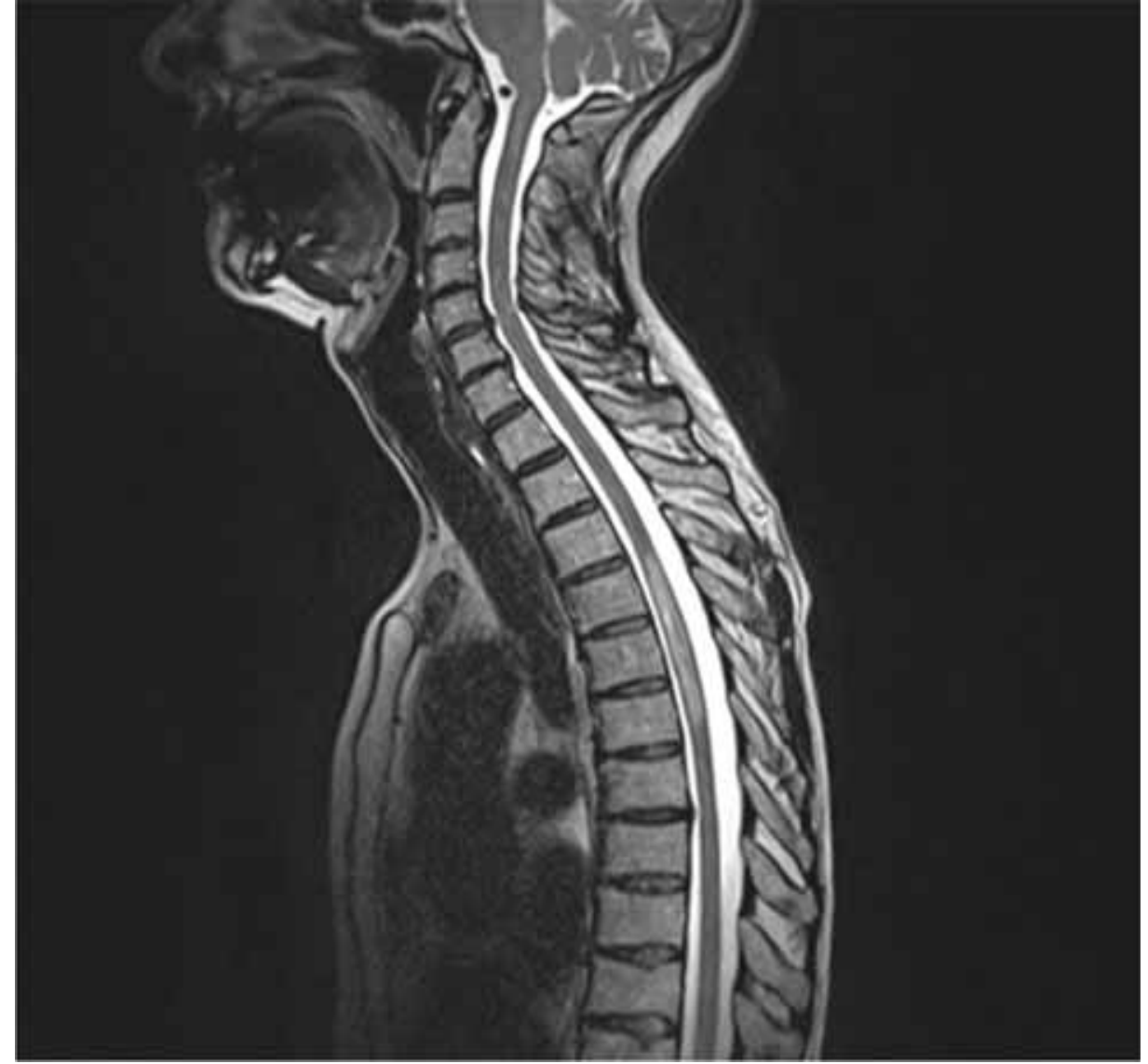

FIGURE 1. Spinal cord MRIsagital section showing increased T2 signal extending from the $T 3$ vertebral body to $T 5$, in a case of NMOSD manifested as longitudinally extensive transverse meylitis relapses occurred, and the AQP4-IgG titre decreased to its lowest value, reaching 3 times the upper reference range limit.

\section{DISCUSSION}

We report a case of NMOSD occurring after the successful treatment of chronic HCV hepatitis with pegylated interferon alpha and ribavirin. Chronic $\mathrm{HCV}$ infection is known to trigger or worsen pre-existing autoimmune diseases (5), while the administration of interferon alpha, the most widely used drug for its treatment, may be associated with deleterious responses and exacerbations of Th17-mediated autoimmune conditions, such as NMOSD and Sjögren syndrome (6). NMOSD cases with onset during interferon alpha administration for $\mathrm{HCV}$ (7), as well as the occurrence of
NMOSD in untreated chronic hepatitis $\mathrm{C}$ patients (8), have been reported. In our case, considering that sustained undetectable HCV viremia was obtained early after treatment initiation, and that the first symptoms suggestive for NMOSD begun several months after the end of the treatment, one could speculate, as already advanced by others (6), that interferon alpha may have had a disease mitigating effect. The present case exemplifies a good correlation between AQP4-IgGs, NMOSD activity, and the therapeutic interventions, adding to the already available empirical knowledge $(1,2)$, and suggesting that periodic plasma exchanges could be considered as a viable alternative for preventing relapses, if complications or associated diseases preclude the administration of immunosuppressant drugs.

\section{REFERENCES}

1. Wingerchuk D.M. et al. International consensus diagnostic criteria for neuromyelitis optica spectrum disorders. Neurology, 2015. 85(2): p. 177-89.

2. Jasiak-Zatonska M. et al. The Immunology of Neuromyelitis Optica-Current Knowledge, Clinical Implications, Controversies and Future Perspectives. Int J Mol Sci, 2016. 17(3): p. 273. 
3. Sellner J. et al. EFNS guidelines on diagnosis and management of neuromyelitis optica. Eur J Neurol, 2010. 17(8): p. 1019-32.

4. Pittock S.J. et al. Neuromyelitis optica and non organ-specific autoimmunity. Arch Neurol, 2008. 65(1): p. 78-83.

5. Ramos-Casals M. et al. Systemic autoimmune diseases in patients with hepatitis C virus infection: characterization of 1020 cases (The HISPAMEC Registry). J Rheumatol, 2009. 36(7): p. 1442-8.

6. Oji S. et al. Experimental Neuromyelitis Optica Induces a Type I Interferon Signature in the Spinal Cord. PLoS One, 2016. 11(3): p. e0151244.
7. Mangioni D. et al. A case of classic neuromyelitis optica (Devic's syndrome) triggered by pegylated-interferon alpha. BMC Pharmacol Toxicol, 2014. 15: p. 56.

8. Earnest B.S. et al. Chronic hepatitis $C$ infection associated with neuromyelitis optica and antinuclear antibodies. J Assoc Physicians India, 2010. 58: p. 118-20. 